\title{
Motor, Somatosensory, Viscerosensory and Metabolic Impairments in a Heterozygous Female Rat Model of Rett Syndrome
}

\author{
Aritra Bhattacherjee ${ }^{1,2}$, Michelle K. Winter ${ }^{2}$, Linda S. Eggimann ${ }^{2}$, Ying Mu ${ }^{1,2}$, \\ Sumedha Gunewardena ${ }^{1,2}$, Zhaohui Liao ${ }^{1,2}$, Julie A. Christianson ${ }^{2,3} \mathbb{E}$ and Peter G. Smith ${ }^{1,2, *}$ \\ 1 Department of Molecular and Integrative Physiology, University of Kansas Medical Center, \\ Kansas City, KS 66160, USA; Aritra.Bhattacherjee@childrens.harvard.edu (A.B.); mu2@uthsc.edu (Y.M.); \\ sgunewardena@kumc.edu (S.G.); zliao@kumc.edu (Z.L.) \\ 2 Kansas Intellectual and Developmental Disabilities Research Center, University of Kansas Medical Center, \\ Kansas City, KS 66160, USA; mwinter2@kumc.edu (M.K.W.); leggimann@kumc.edu (L.S.E.); \\ jchristianson@kumc.edu (J.A.C.) \\ 3 Department of Anatomy and Cell Biology, University of Kansas Medical Center, Kansas City, KS 66160, USA \\ * Correspondence: psmith@kumc.edu; Tel.: +1-913-588-5970; Fax: +1-913-588-5677
}

Received: 3 October 2017; Accepted: 22 December 2017; Published: 29 December 2017

\begin{abstract}
Rett Syndrome (RTT), an autism-related disorder caused by mutation of the X-linked Methyl CpG-binding Protein 2 (MECP2) gene, is characterized by severe cognitive and intellectual deficits. While cognitive deficits are well-documented in humans and rodent models, impairments of sensory, motor and metabolic functions also occur but remain poorly understood. To better understand non-cognitive deficits in RTT, we studied female rats heterozygous for Mecp 2 mutation $\left(M e c p 2^{-/ x}\right)$; unlike commonly used male Mecp $2^{-l y}$ rodent models, this more closely approximates human RTT where males rarely survive. Mecp $2^{-/ x}$ rats showed rapid, progressive decline of motor coordination through six months of age as assessed by rotarod performance, accompanied by deficits in gait and posture. Mecp $2^{-/ x}$ rats were hyper-responsive to noxious pressure and cold, but showed visceral hyposensitivity when tested by colorectal distension. Mecp $2^{-/ x}$ rats ate less, drank more, and had more body fat resulting in increased weight gain. Our findings reveal an array of progressive non-cognitive deficits in this rat model that are likely to contribute to the compromised quality of life that characterizes RTT.
\end{abstract}

Keywords: autism spectrum disorder; behavior; feeding

\section{Introduction}

Autism spectrum disorders (ASDs) and related ASD-like disorders are recognized primarily by cognitive and intellectual deficits. Rett syndrome (RTT) is one of the most severe autism-like disorders affecting, one in 10,000 female births. It is caused by mutation of the X-linked gene MECP2, which is highly expressed in neurons and regulates chromatin organization and global gene transcription [1-3]. RTT is associated with severe cognitive, psychomotor and intellectual disabilities [4,5]. Intellectual and cognitive defects in RTT have been extensively studied in humans as well as animal models [6]. Cognitive deficits such as impaired sociability, anxiety, nest building, contextual fear conditioning, memory function and behavioral regression are prominent in rodent models [7-14], and studies using these MECP2 knockout animals have contributed much to our current mechanistic understanding of RTT [15].

RTT subjects are also reported to exhibit profound deficits in a wide range of basic physiological parameters including sensory perception, motor coordination, respiration and GI function, as well as 
metabolic processes [4,5,16-20]. These dysfunctions contribute to reduced quality of life and health, and frequently aggravate behavioral symptoms. The nature of these dysfunctions and the time course over which they appear, however, remain poorly understood. It has been particularly difficult to document these features in human subjects, given that communication skills are severely compromised by this disability. Unlike other ASDs whose origins involve complex gene-environment interactions or are unidentified, RTT is monogenic and mutation of the MECP2 gene alone in animal models recapitulates many features of the human disease. This provides an opportunity to systematically study onset, nature and progression of non-cognitive dysfunctions [4,6] in RTT.

RTT in humans is essentially a female disorder. While both males and females may harbor Mecp2 mutations of the $\mathrm{X}$ chromosome, males born with the mutation typically die within a few months due to severe encephalopathy [4]. Hence, more than $90 \%$ of surviving human RTT patients are heterozygous females with Mecp 2 mutation (Mecp $2^{-1 x}$ ) [4]. Females with RTT display mosaicism in the expression of the Mecp 2 mutation; $\mathrm{X}$ chromosome inactivation leads to normal expression of Mecp 2 in some cells while others lack expression. However, animal studies to date have relied predominantly on male hemizygous rodents (Mecp $\left.2^{-/ y}\right)$. Male rodent knockouts $(\mathrm{KO})$ survive after birth but display a more severe phenotype that fails to capture the regressive stages of the disease that occur in humans and show a reduced lifespan of up to 2 months $[4,6,15]$, whereas human females can typically live to middle age. Accordingly, limitations of the standard male rodent model of RTT make it desirable to explore models that may more faithfully capture features of the disorder.

In the current study, we used a heterozygous female rat $\left(\mathrm{Mecp}^{-/ x}\right)$ that more closely approximates human RTT, and studied longitudinally a wide range of non-cognitive functions.

\section{Results}

\subsection{Motor Function}

Motor deficits are one of the most prominent non-cognitive impairments in girls with RTT [4]. We assessed motor coordination by rotarod performance test in $M e c p 2^{-1 x}$ rats longitudinally over time. Starting at four weeks of age, we measured biweekly the ability of $M e c p 2^{-/ x}$ and $M e c p 2^{x / x}$ rats to remain on the rotarod during a progressively increasing rate of rotation. There was no difference between groups at four weeks, and Mecp $2^{x / x}$ showed consistent performance through 22 weeks (Figure 1A). However, biweekly measurements of $M e c p 2^{-/ x}$ showed significant declines at 6-10 weeks, plateauing with severely impaired performance at around 16 weeks (Figure 1A and Table 1).

To gain further insight into the mechanics of locomotor dysfunction, we analyzed gait at 22 weeks, after the decline in rotarod performance had reached a stable plateau. DigiGait parameters for gait analysis can be broken down into the major phases of the step cycle, "brake", "swing" and "propel" [21]. "Brake" is the interval from the first contact with the surface to peak stance, "Propel" is the phase from peak until end of contact, and the transitional phase during which a paw is not in contact and extends forward is referred to as "swing". In the forepaw step cycle, brake and swing times were increased, whereas time spent in the propel phase was decreased relative to wild type (WT) rats (Figure 1B). In contrast, the hind paw step cycle did not show statistically significant differences (not shown); however, hind limb stance width, which is the distance between the centers of the hind paws at peak stance, was increased, reflecting postural deficits (Figure 1C). Mecp $2^{-/ x}$ also showed forepaw ataxia; the ataxia coefficient (maximum stride length minus minimum stride length) in $M e c p 2^{x / x}=1.6 \pm 0.093$, and $M e c p 2^{-/ x}=1.24 \pm 0.129(p=0.038)$.

Because alterations in motor control of stepping could impact ambulation, we assessed free ambulatory behavior of the rats. Measurements over a $24 \mathrm{~h}$ period at 22 weeks revealed a moderate decline of diurnal activity. However, there was no difference in nocturnal activity in WT and mutant rats (Figure 1D). 

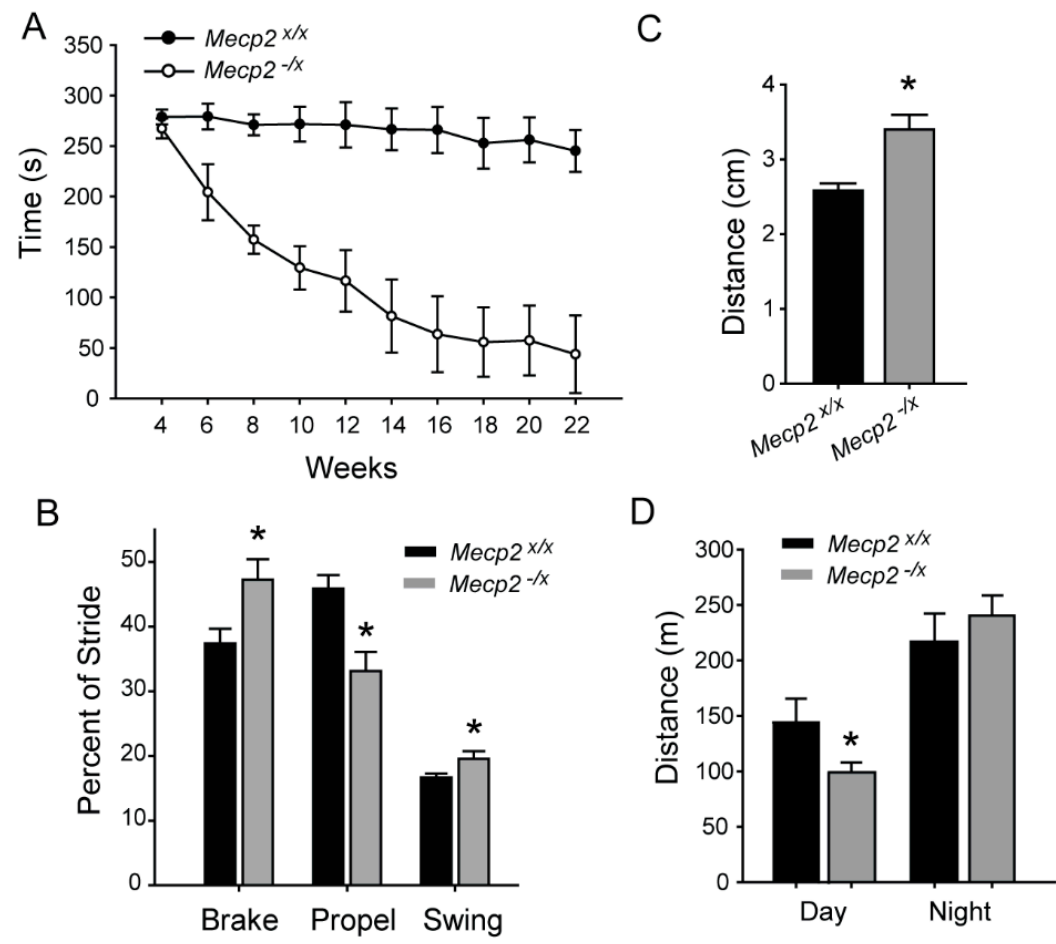

Figure 1. Motor impairments in RTT. (A) Biweekly measurements of Rota-rod performance in WT $M e c p 2^{x / x}$ and Mecp $2^{-1 x}$ rats. Two-way repeated measure Analysis of Variance (ANOVA) reveals significant difference at all ages starting from six weeks $(p<0.001)$; Holm-Sidak post hoc comparisons ( $n=10$ per group). See Table 1 for statistical details; (B) Forepaw gait analysis using the DigiGait system in WT and Mecp $2^{-1 x}$. Comparison by $t$-test reveals significant difference between the two groups in Brake $(* p=0.021)$, Propel $(* p=0.0027)$ and Swing $\left({ }^{*} p=0.024\right)$, expressed as percentage of Stride, as measured from the forepaw; (C) Hind paw stance width of the mutant animals was greater ${ }^{*} p=0.0009, t$-test). $n: M e c p 2^{x / x}=10$ and $M e c p 2^{-/ x}=6$ for $(\mathbf{B})$ and $(\mathbf{C}) ; 4 M e c p 2^{-/ x}$ animals were excluded because they could not stay on the DigiGait treadmill; (D) Difference in distance traveled in a 24-h period by the rats, split between the day and night cycle. Mutant rats are hypoactive during the day $(* p=0.037)$, but show no difference relative to wildtype at night $(p=0.434)(n=\mathrm{WT}=5$ and Mecp $\left.2^{-/ x}=10\right)$. All graphs represent Mean \pm SEM.

\subsection{Sensory Function}

Because studies in both humans and male rodent models of RTT suggest that sensory function is altered $[16,17,22,23]$, we studied responses to somatosensory stimuli including mechanical pressure, noxious heat and noxious cold. Behavioral responses to these stimuli were measured longitudinally from four through 22 weeks of age.

Mechanical sensitivity was tested using graded von Frey monofilaments applied to the hind foot paw to determine the threshold for withdrawal response. Thresholds were generally lower in younger rats and no significant difference could be resolved between wild type and heterozygotes at four and six weeks of age. However, while thresholds rose beginning at eight weeks in Mecp $2^{x / x}$ as part of normal developmental course, $M e c p 2^{-/ x}$ rats remained hypersensitive throughout the adult life with little change of threshold through 22 weeks (Figure 2A and Table 1).

Response to noxious cold was tested by recording the temperature for paw withdrawal on a cold plate. A general tendency toward higher sensitivity to noxious cold existed in $M e c p 2^{-/ x}$ rats from the beginning; however, significant differences became prominent from eight weeks of age that grew more severe over the subsequent weeks of testing (Figure 2B and Table 1). 
Table 1. Summary of statistical analyses for two-way repeated measure ANOVAs.

\begin{tabular}{ccccc}
\hline Test & Source of Variation & DF & F & P \\
\hline \multirow{3}{*}{ Body Weight } & Genotype & 1 & 12.487 & 0.002 \\
& Weeks & 9 & 310.219 & $<0.001$ \\
& Genotype $\times$ Weeks & 9 & 6.613 & $<0.001$ \\
\hline \multirow{3}{*}{ Rotarod } & Genotype & 1 & 83.617 & $<0.001$ \\
& Weeks & 9 & 16.738 & $<0.001$ \\
& Genotype $\times$ Weeks & 9 & 8.143 & $<0.001$ \\
\hline \multirow{3}{*}{ Von Frey } & Genotype & 1 & 21.737 & $<0.001$ \\
& Weeks & 9 & 3.649 & $<0.001$ \\
& Genotype $\times$ Weeks & 9 & 0.281 & 0.979 \\
\hline \multirow{3}{*}{ Cold Plate } & Genotype & 1 & 36.002 & $<0.001$ \\
& Weeks & 9 & 2.266 & 0.021 \\
& Genotype $\times$ Weeks & 9 & 0.780 & 0.635 \\
\hline \multirow{2}{*}{ VMR } & Genotype & 1 & 6.514 & 0.021 \\
& Pressure & 3 & 33.405 & $<0.001$ \\
& Genotype $\times$ Pressure & 3 & 4.600 & 0.007 \\
\hline
\end{tabular}

A

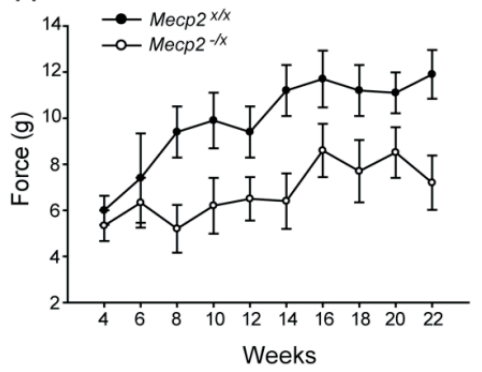

B

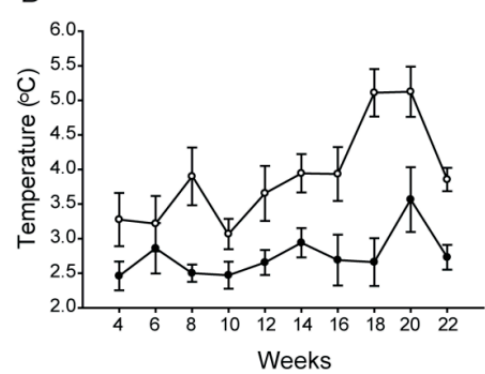

C

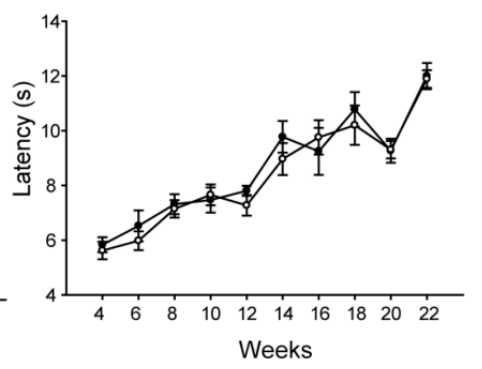

Figure 2. Somatic sensitivity in the RTT rat model. (A) Biweekly measurements using von Frey monofilaments reveals mechanical hypersensitivity in $M e c p 2^{-1 x}$ rats at all ages starting from eight weeks (Holm-Sidak post hoc comparisons, $p<0.001$ at 8-22 weeks); (B). Biweekly measurements of noxious cold sensitivity using the cold plate reveals hypersensitivity in $M e c p 2^{-/ x}$ rats at all ages starting from 8 weeks (two-way repeated measure ANOVA, Holm-Sidak post hoc comparisons, $p<0.001$ ); (C) Noxious heat sensitivity tested using the Hargreaves Paw Thermal Analgesiometer. No difference between the two groups registered during the entire testing period. ( $n$ : WT $\left.=10, M e c p 2^{-/ x}=14\right)$. Further details of statistical parameters for $(\mathbf{A}, \mathbf{B})$ in Table 1. All graphs represent Mean \pm SEM.

Response to noxious heat was tested by using the paw thermal analgesiometer, where a light beam is directed to the Plexiglas surface immediately beneath a hind paw and the latency for foot withdrawal is measured. Both WT and $M e c p 2^{-/ x}$ rats showed increased response latencies with age, consistent with a developmental diminution in thermal sensitivity. However, unlike mechanical or cold sensitivity, there were no differences in response latencies to noxious heat between the two groups at any age point (Figure 2C).

The gastrointestinal system is imbued with stretch receptors that monitor pressure and elicit reflex contractile responses in the surrounding musculature [24]. Because there are reports that RTT patients show visceral discomfort under certain conditions, we assessed whether responses to colorectal distension [24] are affected in the Mecp $2^{-/ x}$ rat. (Figure 3A). In response to applied colorectal pressures from 20 to $80 \mathrm{~mm} \mathrm{Hg}$, the visceromotor response (VMR) increased in both WT and Mecp2 $2^{-/ x}$ animals. However, the heterozygous animals showed reduced VMR relative to WT rats at pressures from 40 to $80 \mathrm{~mm} \mathrm{Hg}$ (Figure 3B and Table 1), consistent with visceral hyposensitivity. 
A

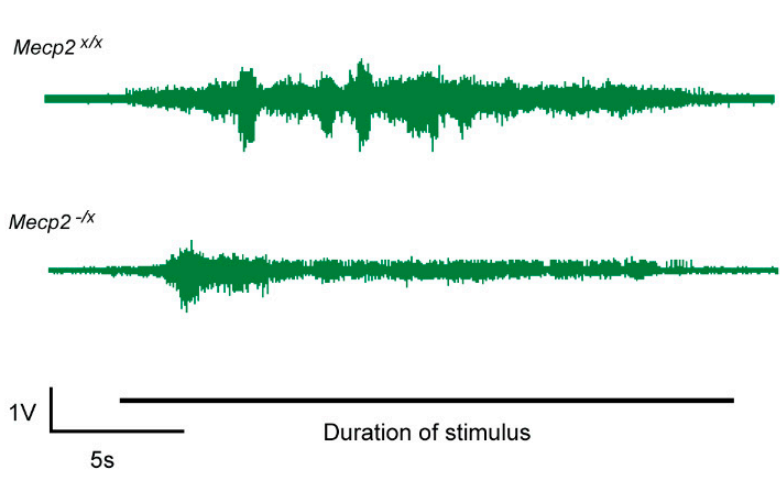

B

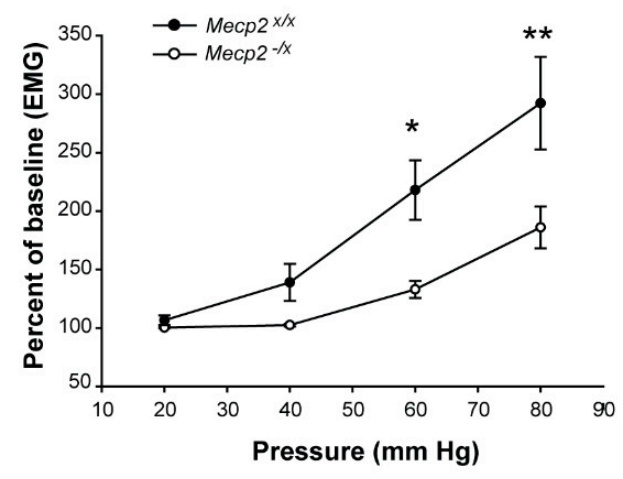

Figure 3. Visceral sensitivity in the RTT rat model. (A) Representative electromyography (EMG) recordings of visceromotor response in abdominal muscles of $M e c p 2^{x / x}$ and $M e c p 2^{-1 x}$ rats for colorectal distension at $80 \mathrm{~mm} \mathrm{Hg}$; (B) Comparison of visceromotor responses expressed as activity per unit time, in 22-week-old rats reveal hyposensitivity to colorectal distension at 40,60 and $80 \mathrm{~mm} \mathrm{Hg}$ pressures. EMG/s in $M e c p 2^{-/ x}$ rats is significantly lower at $60 \mathrm{~mm} \mathrm{Hg}\left(^{*} p=0.006\right)$ and $80 \mathrm{~mm} \mathrm{Hg}\left({ }^{* *} p<0.001\right)$ pressures (two-way repeated measure ANOVA, Holm-Sidak Posthoc Analysis; $n$ : $\mathrm{WT}=10$, Mecp $2^{-/ x}=8$. See Table 1 for details. Graph represents Mean \pm SEM.

\subsection{Metabolic Indicators}

Metabolic dysfunction is increasingly associated with autism spectrum disorders [25,26]. We therefore assessed indices of metabolic activity to determine if female rats heterozygous for the Mecp 2 mutation showed phenotypes consistent with this type of abnormality. At postnatal weeks 4-6, body weights of $M e c p 2^{-/ x}$ and $M e c p 2^{x / x}$ rats were comparable. Beginning at eight weeks of age, however, $M e c p 2^{-1 x}$ rats were heavier and the difference increased with time throughout the 22 weeks of the study (Figure $4 \mathrm{~A}$ ).

We assessed the proximate cause of the increased body weight by analyzing body mass composition at 22 weeks using an Echo-MRI. We found no difference in the lean body mass or total body water content between the two groups (Figure 4B,C). However, total body fat was elevated approximately three-fold in the $M e c p 2^{-/ x}$ relative to the $M e c p 2^{x / x}$ rats (Figure 4D).

To assess whether there may be strain-dependent differences in caloric intake, we measured food consumption over a 48 -h period in a metabolic chamber. In WT rats, food was consumed in the diurnal phase although nocturnal consumption was significantly greater (Figure $4 \mathrm{E}$ ). Surprisingly, Mecp $2^{-/ x}$ rats ate substantially less food (Figure $4 \mathrm{E}$ ). Night time consumption was reduced by approximately $1 / 3$, whereas consumption during the daytime was only about $30 \%$ of WT controls.

Water intake was also affected by the Mecp 2 mutation. WT rats showed similar water consumption in both the diurnal and nocturnal phases (Figure $4 \mathrm{~F}$ ). Daytime drinking in $M e c p 2^{-/ x}$ rats was similar to WT; however, they drank approximately twice as much water at night relative to WT rats (Figure 4F).

To assess whether changes in metabolic rate may help to explain why Mecp $2^{-/ x}$ rats gain weight and fat mass despite lower food consumption, we assessed a range of metabolism-associated parameters (Table 2). Mecp $2^{-/ x}$ rats showed consistently lower metabolic values, with a significantly lower respiratory quotient detected during the light phase of the diurnal cycle. 
A
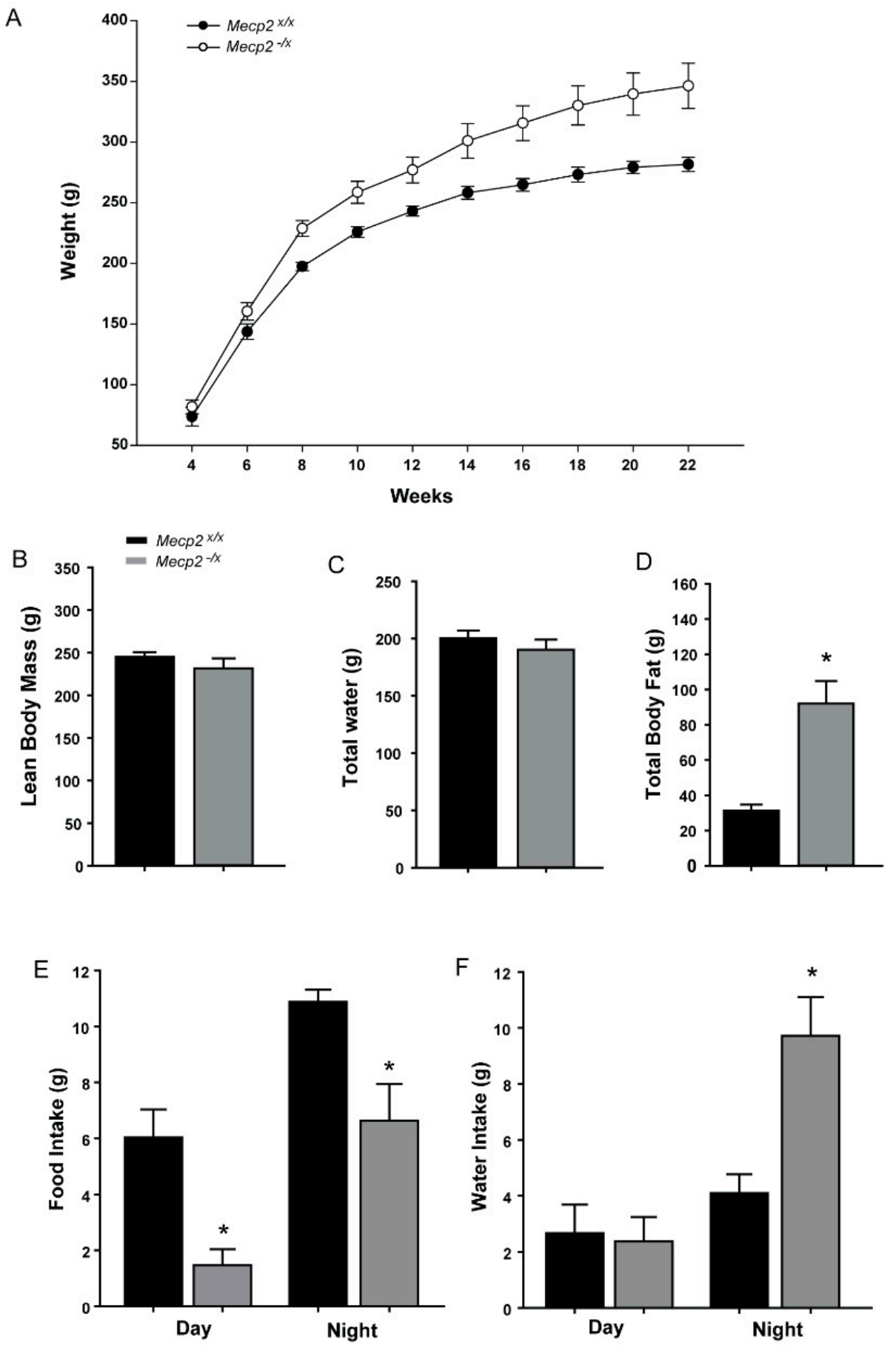

Figure 4. Abnormal weight gain and calorie intake in the RTT rat model. (A) Biweekly weight measurements from $M e c p 2^{x / x}$ and $M e c p 2^{-1 x}$ rats reveal a significantly increased body weight starting from eight weeks of age ( $p<0.001$, two-way repeated measure ANOVA with Holmes-Sidak posthoc analysis; see Table 1 for statistical details); (B-D) Comparison of body mass composition between $M e c p 2^{x / x}$ and $M e c p 2^{-/ x}$ rats by Echo-MRI analysis. No difference in lean body mass (B) or total water content (C) detected between the two groups. Body fat levels were highly elevated in the Mecp $2^{-1 x}$; (D) $p=0.0001$; (E) Food intake comparison between the two groups revealed lower consumption by the $M e c p 2^{-/ x}$ rat both during day $(p=0.001)$ and night $(p=0.044)(t$-tests); (F) Water intake compared between the two groups of rats. No diurnal difference, but nocturnal consumption is highly increased in $M e c p 2^{-1 x}$ rats $\left(p=0.016, t\right.$-test. $n$ : WT $\left.=5, M e c p 2^{-/ x}=10\right)$. All graphs represent Mean $\pm \mathrm{SEM}$. 
Table 2. Metabolic indices measured by calorimetry during light and dark phases over a 48-h test period in wild type (WT) and heterozygous (Het) rats. VO2 = oxygen consumed as $\mathrm{O} 2 \mathrm{~mL} / \mathrm{min}$. $\mathrm{VCO} 2$ = carbon dioxide produced as $\mathrm{mL} / \mathrm{min}$. $\mathrm{RQ}=$ Respiratory Quotient obtained as VCO2/VO2. All measurements are from a single rat per cage.

\begin{tabular}{cccccc}
\hline & \multicolumn{7}{c}{ Light } \\
\hline Parameters & WT Mean & WT SEM & Het Mean & Het SEM & $\boldsymbol{t}$-Test $\boldsymbol{p}$-Value \\
\hline Average Energy Expenditure & 1.85 & 0.04 & 1.67 & 0.09 & 0.199 \\
Total Energy Expenditure & 25.96 & 0.56 & 23.43 & 1.27 & 0.199 \\
Average VO2 & 6.41 & 0.14 & 5.88 & 0.31 & 0.26 \\
Average VCO2 & 5.09 & 0.16 & 4.28 & 0.28 & 0.073 \\
Average RQ & 0.79 & 0.02 & 0.72 & 0.02 & 0.03 \\
\hline & & Dark & & & \\
\hline Parameters & WT Mean & WT SEM & Het Mean & Het SEM & $\boldsymbol{t}$-Test $p$-Value \\
\hline Average Energy Expenditure & 2.29 & 0.03 & 2.01 & 0.12 & 0.138 \\
Total Energy Expenditure & 22.85 & 0.31 & 20.09 & 1.2 & 0.138 \\
Average VO2 & 7.86 & 0.13 & 7.01 & 0.41 & 0.172 \\
Average VCO2 & 6.42 & 0.11 & 5.3 & 0.38 & 0.064 \\
Average RQ & 0.82 & 0.02 & 0.75 & 0.02 & 0.054 \\
\hline
\end{tabular}

\subsection{Inter-Relationships among MECP2 Mosaicism and Behavioral and Metabolic Dysfunctions}

Because MECP2 is an X-linked gene, random X-chromosome inactivation means that each RTT female patient (or rat) is a mosaic of MECP2 expressing and null cells, and the degree of mosaicism among different individuals can vary. We reasoned that the degree of mosaicism, or heterozygosity, may correlate with disease severity. We analyzed $M e c p 2^{-/ x}$ rats for their degree of mosaicism in peripheral blood T-cells as a representative cell population that is easily isolated. Blood samples were drawn from tail veins of the rats and peripheral blood mononuclear cells (PBMNCs) isolated. PBMNCs were fixed and stained for Mecp 2 and the T-cell marker CD3. Fluorescence assisted cell sorting (FACS) analysis of these samples revealed the percentage of T-cells expressing Mecp2. We were unable to detect any significant correlation between degree of T-cell mosaicism and behavioral or metabolic dysfunction in any of the measured parameters. Similarly, when we assessed the relationships among different behavioral and metabolic parameters, strong correlations were not evident with the exception of deficits in rotarod performance correlating with a decrease in the swing phase of the step cycle (Table 3).

Table 3. Summary of regression analyses performed among behavioral measurements in $M e c p 2^{-/ x}$ rats.

\begin{tabular}{|c|c|c|c|c|}
\hline Parameters Compared & $\mathbf{R}^{2}$ & Adjusted $\mathbf{R}^{2}$ & $\begin{array}{l}\text { F-Statistic vs. } \\
\text { Constant Model }\end{array}$ & $p$-Value \\
\hline Weight gain rate and day time food intake & 0.0152 & -0.108 & 0.124 & 0.734 \\
\hline Weight gain rate and day time water intake & 0.0405 & -0.0794 & 0.338 & 0.577 \\
\hline Weight gain rate and night time water intake & $5.73 \times 10^{-6}$ & -0.125 & $4.58 \times 10^{-5}$ & 0.995 \\
\hline von Frey and Brake & 0.195 & -0.00572 & 0.972 & 0.38 \\
\hline Sensitivity ( $\%$ withdrawal) to a $4 \mathrm{mg}$ filament and brake & 0.564 & 0.456 & 5.18 & 0.0851 \\
\hline Sensitivity (\% withdrawal) to a $4 \mathrm{mg}$ filament and Propel & 0.514 & 0.393 & 4.23 & 0.109 \\
\hline Sensitivity (\% withdrawal) to a $4 \mathrm{mg}$ filament and Swing & 0.0482 & -0.19 & 0.202 & 0.676 \\
\hline Rotarod and Brake & 0.204 & 0.00471 & 1.02 & 0.369 \\
\hline Rotarod and Propel & 0.0255 & -0.218 & 0.105 & 0.762 \\
\hline Rotarod and Swing & 0.706 & 0.633 & 9.62 & 0.0362 \\
\hline
\end{tabular}




\section{Discussion}

This work demonstrates the feasibility of using heterozygous female rats to study progressive non-cognitive pathologies associated with RTT. While homozygous male MECP2 mutant rodents have been used for most RTT animal model studies to date, almost all human patients are heterozygous females. Our observations show that heterozygous female animals develop impairments similar to those seen with homozygous males [22], albeit with later onset, and potentially greater variability that is consistent with human observations. Moreover, the rat has proven advantageous as a model for resolving the longitudinal progression of disease symptoms. Behavioral responses in rat are robust and provide a broad dynamic range to detect regressive changes over time. In fact, before the advent of mouse genetics, the rat was a highly preferred model for study of sensory, motor and cognitive behaviors [27].

Consistent with the human disorder [28], motor impairments emerged as one of the most significant deficits in the $M e c p 2^{-1 x}$ rats. Motor behavioral deficits have been reported previously in $M e c p 2^{-l y}$ mice at up to 60 days post-natal, but the time course is not well established $[22,23,29,30]$. In the current study, we followed female subjects for longer than five months and found that motor function declines steadily through about four months of age, which may be consistent with the progressive decline with age observed in the late motor deterioration stage in patients with RTT. Interestingly, voluntary ambulatory behavior remained largely preserved throughout the study period; even when $M e c p 2^{-1 x}$ rats could barely remain on the rotarod, there was no difference of night time voluntary activity and only a moderate decline in diurnal activity, which may suggest that central structures initiating movement may not be affected. On the other hand, poor rotarod performance did correlate significantly with increased swing phase of the step cycle, suggesting that the increased time required to move the foot into the braking position may have led to instability and discoordination.

A curious feature of the motor dysfunction is the rostro-caudal asymmetry of the effect, where the forelimbs are disproportionately affected. This would seem to be consistent with prior reports in heterozygous Mecp2-deficient mice [30]. The Mecp2-308 female mice show defects in purposeful forelimb use as evidenced by impaired handling of nesting material, food and novel environment exploration [31]. Deficient forelimb movement and repetitive patterns is also a key feature of human RTT [32-34]. Hence, a greater deficit in forelimb motor function appears to be consistent across male mouse and female rat RTT models as well as in human subjects.

Somatosensory deficits are widespread in ASD [35,36] and abnormal sensory perception as well as indications of pain in RTT patients have been reported by parents and care-givers $[16,17]$. Nonetheless, it has been challenging to document the exact nature and progression of sensory deficits in patients, which is especially problematic in RTT owing to deficits in verbal communication abilities. Using Mecp $2^{-l y}$ rodent models, we and others found somatosensory deficits associated with Mecp 2 mutation which appears to be cell-autonomous to primary sensory neurons of the dorsal root ganglion [22,23]. Hence, deletion of Mecp2 appears to result in altered transcription within the ganglion that directly affects sensory neurons through effects at the levels of the peripheral and central terminals [22]. Consistent with our findings in male $M e c p 2^{-l y}$ rats, $M е с p 2^{-/ x}$ female rats showed marked hypersensitivity to mechanical and noxious cold stimuli [22] although the onset was marginally delayed (eight weeks vs. four weeks in $M e c p 2^{-l y}$ ) but sustained thereafter. In contrast to $M e c p 2^{-l y}$ rats, thermal hypoalgesia was not observed in $M e c p 2^{-/ x}$ rats.

One question that remains unanswered is whether other behavioral deficits occur secondary to somatosensory disturbances. In light of the increased mechanical sensitivity associated with Mecp2 deletion, it is possible that this could contribute to reduced motor performance. Hence, it is possible that the step cycle may be impacted by 'guarding', where animals preferentially spend less time in the propel phase where the increased force associated with propulsion could be perceived as painful. Similarly, while we know that sensory nociceptor [22] and low threshold tactile receptor [23] neuronal functions are altered, it is likely that other types of sensory function may also be disturbed. Accordingly, it is possible that proprioception may also be affected and, if so, this could contribute to alterations in 
step cycle and posture that occur in these animals. Further investigation will be necessary to elucidate the extent to which motor deficits occur secondary to sensory dysfunction.

This study also provides evidence that visceral perception is disrupted in this female RTT rat model. In human RTT patients, gastrointestinal and bowel related problems are prominent [20,37]. There is strong evidence that patients with RTT experience significant gastrointestinal dysmotility leading to gastroesophageal reflux, constipation, bloating, with resultant distress and frequent abdominal pain [37]. Our findings point to colonic hyposensitivity and/or hyporeflexia as a prominent component of the RTT rat model phenotype. Balloon distension of the colon resulted in a much-diminished abdominal muscular activation, which would be consistent with dysmotility. Visceral function and sensitivity are regulated by complex circuits involving intrinsic enteric neuronal plexuses as well as extrinsic contributions from the autonomic and nervous system and dorsal root ganglia [38-42]. Dysregulation of autonomic functions has been reported in RTT [43-45], although Mecp2 is widely expressed in all neuronal and target cells such that the etiology of the visceral dysfunction remains uncertain. Nonetheless, the $M e c p 2^{-1 x}$ rat should prove to be a useful model in helping to define mechanisms of RTT visceral dysfunction.

Many pervasive developmental disorders including autism are associated with alterations in metabolism [25,26]. However, prior studies using mouse models failed to reveal a systematic trend in metabolic disruption [15]. This may be attributable to the short duration of studies, use of null male mice which are underweight and survive only to two months of age, and/or interactions between the mutation and the genetic background of the different strains. Some studies of Mecp 2 mouse models have reported altered body weight, with both increases and decreases observed and occurring in a potentially strain-specific manner [46-48]. Mecp2 deletion restricted to the hypothalamus results in increased weight gain in association with increased food intake [46]. Our findings of increased weight gain in association with reduced food intake suggest that possibly other brain regions associated with satiety may be differentially affected in the different models, or that perhaps there is a more profound metabolic disturbance in the heterozygous female rat. Indeed, the Mecp $2^{-/ x}$ rat also showed abnormal fat accumulation despite significantly lower food intake and largely comparable activity levels, consistent with severe metabolic dysfunction. This is also consistent with some missense and late truncating Mecp 2 mutations that give rise to a RTT variant known as the Zappella, where subjects are overweight [4,49]. Humans with RTT show elevated total cholesterol and LDL levels in plasma [50], and Mecp $2^{-/ y}$ mice exhibit defective cholesterol metabolism in brain and liver [51]. It is unclear whether the increased body fat promotes a systemic pro-inflammatory state that may alter sensory neuron thresholds [52,53], thus contributing to the hypersensitivity we see in this model. It is noteworthy that both maternal fostering and genetic modifiers associated with different background strains have been shown to be strong contributors to behavioral and metabolic phenotype [54,55], and interactions with different mutations might also play a prominent role in determining the metabolic outcome. The molecular mechanisms underlying metabolic dysfunctions in RTT remain to be determined.

\section{Materials and Methods}

\subsection{Animals}

All animal experiments were conducted on a total of 24 female rats and were approved by the KUMC IACUC. Associated protocols used in this study will be provided upon request. The MECP2 knockout rat was generated by a zinc finger nuclease mediated 71 bp deletion in exon 4 of MECP2 gene (Product\# TGRA6090, Sage Labs, Boyertown, PA, USA).

\subsection{Timelines for Measurement}

Table 4 outlines the frequency and time line (by animal age) for tests and measurements administered in this study. 
Table 4. Behavioral testing schedule.

\begin{tabular}{ccc}
\hline Test & Frequency of Measure & Age \\
\hline Rotarod & Biweekly & Weeks 4-22 \\
DigiGait & Once & Week 18 \\
Somatosensory (mechanical, thermal, cold) & Biweekly & Weeks 4-22 \\
Visceral sensitivity & Once & Week 18 \\
Metabolic (food, water, activity) & Once & Week 18 \\
Body mass composition & Once & Week 18 \\
\hline
\end{tabular}

\subsection{Rotarod}

Balance and coordination were tested using an accelerating rotarod (Med Associates Inc., St. Albans, Vermont, USA) with an initial speed of $4 \mathrm{rpm}$ ramping to $40 \mathrm{rpm}$ over $300 \mathrm{~s}$. Rats were placed on the 70-mm diameter rod elevated $27 \mathrm{~cm}$ above the table. The accelerating program was initiated and the latency to fall off the rod was recorded and averaged from three independent trials at least 5 min apart.

\subsection{Gait Analysis}

Fully automated gait analysis was conducted with the DigiGait (Mouse Specifics, Framingham, MA, USA) treadmill at a walking speed of $8.5 \mathrm{~cm} / \mathrm{s}$. The DigiGait system records foot falls of the subject from the ventral aspect and then automates the analysis of over 40 parameters including brake, propel and stride time, coordination, and ataxia coefficient [21].

\subsection{Somatosensory Behavior: Mechanical, Thermal and Cold Sensitivity}

Mechanical sensitivity was analyzed using Siemens Weinstein monofilaments of known, graded force. Animals were placed in individual Plexiglas boxes on an elevated mesh table and allowed to acclimate for $20 \mathrm{~min}$. Following acclimation, six monofilaments ranging from 2 to $15 \mathrm{~g}$ were applied five times to each hind paw at intervals of not less than $2 \mathrm{~min}$. The number of positive responses, as observed by lifting or biting of the hind paw, was recorded. The calibrated force of the monofilament generating a $50 \%$ withdrawal response rate was recorded as the withdrawal threshold.

Thermal sensitivity was measured as withdrawal latency to a fixed 4.85-amp source directed to the paw in a Paw Thermal Stimulator (University of California, San Diego, CA, USA). The glass floor was maintained at $30.0^{\circ} \mathrm{C}$ and 20 min of acclimation was allowed prior to testing. Light beam intensity was set at $4.85 \mathrm{amp}$ and withdrawal latency defined as the time from stimulus onset until the automatic sensor turned the light off in response to paw lifting. Thermal withdrawal latencies of each hind paw were measured in triplicate, with a minimum of $3 \mathrm{~min}$ between trials, and were averaged.

Cold sensitivity was measured using a Cold Plate Analgesia Meter (IITC Life Science Inc., Los Angeles, CA, USA). Rats were acclimated for five min at $25^{\circ} \mathrm{C}$, and thermal plate temperature was decreased at a ramp rate of $10^{\circ} \mathrm{C}$ per min. Upon observing overt nociceptive behavior such as lifting, biting or licking of the hind paws, the thermal plate was immediately returned to $25^{\circ} \mathrm{C}$. Testing was conducted twice with a $5-10 \mathrm{~min}$ interval between trials. The average temperature at which nociceptive behaviors were seen is reported as the withdrawal threshold.

\subsection{Visceral Sensitivity}

Rats were initially anesthetized with $4 \%$ inhaled isoflurane followed by continually-administered $1.5-3 \%$ inhaled isoflurane to maintain anesthesia. A small, catheterized $(0.5 \mathrm{~cm} \times 4 \mathrm{~cm})$ latex or nitrile balloon was inserted into the distal colon. The rat was then put on a $37^{\circ} \mathrm{C}$ heating pad under continuous $1.5 \%$ isoflurane anesthesia through nose cone and the balloon was distended for $20 \mathrm{~s}$ to $20,40,60$ and $80 \mathrm{~mm} \mathrm{Hg}$ (each pressure in triplicate with 4 min inter-trial intervals) and the electromyographic activity of the abdominal musculature were measured by thin silver electrode wires 
implanted acutely into the abdominal muscle on either side of the abdominal midline; the ground was attached to the tail. The VMR was quantified by measuring the area under the curve of the entire distension period divided by the duration of distension and expressed as a percentage of baseline EMG activity [24].

\subsection{Food, Water and Activity Monitoring (Metabolic Cage)}

Rats were placed in indirect calorimetry home cages (Sable Systems, Las Vegas, NV, USA) and were preconditioned prior to collection of data to eliminate effects of the novel environment. The gas from the cages is sampled each second for $\mathrm{O}_{2}, \mathrm{CO}_{2}$, and $\mathrm{H}_{2} \mathrm{O}$ content. Food, water and animal habitats are placed on weight sensors and movement of the subject is measured with external infrared beams. Fully automated software calculates activity (free ambulation) respiratory quotient, distance traveled, and others.

\subsection{Body Mass Composition}

Lean and fat mass was analyzed with the EchoMRI Body Composition Analyzer (EchoMRI LLC, Houston, TX, USA). Briefly, unanesthetized rats are placed in the analyzer for approximately $60 \mathrm{~s}$ and proprietary NMR-MRI technology is used to calculate fat, lean, free water and total water of the subject.

\subsection{PBMNC Isolation, Staining and FACS}

About 400-500 $\mu \mathrm{L}$ blood was collected from the tail vein of each $M e c p 2^{-/ x}$ rat into EDTA coated vials. The blood was diluted 1:1 in sterile PBS layered over Percoll and centrifuged. The buffy coat layer containing the peripheral blood mononuclear cells (PBMNCs) was collected and fixed by adding equal volume of $8 \%$ PFA (to make a final $4 \%$ concentration). The cells were washed thrice in PBS and then stained for 20-30 min on ice (wrapped in aluminum foil) with FITC conjugated Anti-CD3 antibody at 1:200 (BD Bioscience, San Jose, CA, USA) to label all T-cells. After a wash, primary anti-Mecp2 (rabbit-anti, Cell Signaling Technology, Danvers, MA, USA) was added and incubated for $1 \mathrm{~h}$. After washes, donkey anti-rabbit Cy3 (Jackson Immuno-research, West Grove, PA, USA) at 1:200 and incubated for $30 \mathrm{~min}$. Following three washes, cells were FACS analyzed to determine the percentage of Mecp2 expressing cells within the CD3 positive population.

\subsection{Stastical Analysis}

The statistical software SigmaPlot (Systat, San Jose, CA, USA) was used to perform analyses reported here. Comparisons of longitudinal measures of behavioral parameters were done by two way repeated measure ANOVA on ranks and using Holms-Sidak for post hoc comparisons. For comparison of two groups and single time point data, unpaired $t$-tests using Student Newman-Keuls were performed. In all cases $p<0.05$ is reported to be significant. All data were reported as Mean $\pm \mathrm{SEM}$, unless stated otherwise.

Acknowledgments: We thank Anuradha Chakrabarty for technical guidance, John Thyfault for assistance with the data from metabolic testing, and the staff of the Kansas Intellectual and Developmental Disabilities Research Center. Funding was provided by NICHD RO1HD049615 with core support from NICHD P30 HD002528 and U54 HD090216, and KUMC's Biomedical Research Training Program.

Author Contributions: Aritra Bhattacherjee and Peter G. Smith conceived and designed the experiments; Aritra Bhattacherjee, Michelle K. Winter, Linda S. Eggimann, Ying Mu and Zhaohui Liao performed the experiments; Aritra Bhattacherjee, Michelle K. Winter and Sumedha Gunewardena analyzed the data; Julie A. Christianson contributed materials and analysis tools; Aritra Bhattacherjee and Peter G. Smith wrote the paper.

Conflicts of Interest: The authors declare no conflict of interest. 


\section{Abbreviations}

$\begin{array}{ll}\text { RTT } & \text { Rett Syndrome } \\ \text { ASD } & \text { Autism spectrum disorder } \\ \text { MECP2 } & \text { Methyl CpG-binding Protein 2 } \\ \text { WT } & \text { Wild type } \\ \text { PBMNC } & \text { Peripheral blood mononuclear cell } \\ \text { EMG } & \text { Electromyography } \\ \text { VMR } & \text { Visceromotor reflex }\end{array}$

\section{References}

1. Amir, R.E.; Van den Veyver, I.B.; Wan, M.; Tran, C.Q.; Francke, U.; Zoghbi, H.Y. Rett syndrome is caused by mutations in X-linked MECP2, encoding methyl-CpG-binding protein 2. Nat. Genet. 1999, 23, 185-188. [PubMed]

2. Chahrour, M.; Jung, S.Y.; Shaw, C.; Zhou, X.; Wong, S.T.; Qin, J.; Zoghbi, H.Y. MeCP2, a key contributor to neurological disease, activates and represses transcription. Science 2008, 320, 1224-1229. [CrossRef] [PubMed]

3. Cheng, T.L.; Qiu, Z. MeCP2: Multifaceted roles in gene regulation and neural development. Neurosci. Bull. 2014, 30, 601-609. [CrossRef] [PubMed]

4. Chahrour, M.; Zoghbi, H.Y. The story of Rett syndrome: From clinic to neurobiology. Neuron 2007, 56, 422-437. [CrossRef] [PubMed]

5. Cianfaglione, R.; Clarke, A.; Kerr, M.; Hastings, R.P.; Oliver, C.; Felce, D. A national survey of Rett syndrome: Age, clinical characteristics, current abilities, and health. Am. J. Med. Genet. Part A 2015, 167, 1493-1500. [CrossRef] [PubMed]

6. Zoghbi, H.Y. MeCP2 dysfunction in humans and mice. J. Child Neurol. 2005, 20, 736-740. [CrossRef] [PubMed]

7. Veeraragavan, S.; Wan, Y.W.; Connolly, D.R.; Hamilton, S.M.; Ward, C.S.; Soriano, S.; Pitcher, M.R.; McGraw, C.M.; Huang, S.G.; Green, J.R.; et al. Loss of MeCP2 in the rat models regression, impaired sociability and transcriptional deficits of Rett syndrome. Hum. Mol. Genet. 2016, 25, 3284-3302. [CrossRef] [PubMed]

8. Moretti, P.; Bouwknecht, J.A.; Teague, R.; Paylor, R.; Zoghbi, H.Y. Abnormalities of social interactions and home-cage behavior in a mouse model of Rett syndrome. Hum. Mol. Genet. 2005, 14, 205-220. [CrossRef] [PubMed]

9. Gemelli, T.; Berton, O.; Nelson, E.D.; Perrotti, L.I.; Jaenisch, R.; Monteggia, L.M. Postnatal loss of methyl-CpG binding protein 2 in the forebrain is sufficient to mediate behavioral aspects of Rett syndrome in mice. Biol. Psychiatry 2006, 59, 468-476. [CrossRef] [PubMed]

10. McGill, B.E.; Bundle, S.F.; Yaylaoglu, M.B.; Carson, J.P.; Thaller, C.; Zoghbi, H.Y. Enhanced anxiety and stress-induced corticosterone release are associated with increased Crh expression in a mouse model of Rett syndrome. Proc. Natl. Acad. Sci. USA 2006, 103, 18267-18272. [CrossRef] [PubMed]

11. Moretti, P.; Levenson, J.M.; Battaglia, F.; Atkinson, R.; Teague, R.; Antalffy, B.; Armstrong, D.; Arancio, O.; Sweatt, J.D.; Zoghbi, H.Y. Learning and memory and synaptic plasticity are impaired in a mouse model of Rett syndrome. J. Neurosci. Off. J. Soc. Neurosci. 2006, 26, 319-327.

12. Santos, M.; Silva-Fernandes, A.; Oliveira, P.; Sousa, N.; Maciel, P. Evidence for abnormal early development in a mouse model of Rett syndrome. Genes Brain Behav. 2007, 6, 277-286. [CrossRef] [PubMed]

13. Shahbazian, M.D.; Young, J.I.; Yuva-Paylor, L.A.; Spencer, C.M.; Antalffy, B.A.; Noebels, J.L.; Armstrong, D.L.; Paylor, R.; Zoghbi, H.Y. Mice with truncated MeCP2 recapitulate many Rett syndrome features and display hyperacetylation of histone H3. Neuron 2002, 35, 243-254. [CrossRef]

14. Stearns, N.A.; Schaevitz, L.R.; Bowling, H.; Nag, N.; Berger, U.V.; Berger-Sweeney, J. Behavioral and anatomical abnormalities in Mecp2 mutant mice: A model for Rett syndrome. Neuroscience 2007, 146, 907-921. [CrossRef] [PubMed]

15. Ricceri, L.; De Filippis, B.; Laviola, G. Mouse models of Rett syndrome: From behavioural phenotyping to preclinical evaluation of new therapeutic approaches. Behav. Pharmacol. 2008, 19, 501-517. [CrossRef] [PubMed] 
16. Symons, F.J.; Byiers, B.; Tervo, R.C.; Beisang, A. Parent-reported pain in Rett syndrome. Clin. J. Pain 2013, 29, 744-746. [CrossRef] [PubMed]

17. Barney, C.C.; Feyma, T.; Beisang, A.; Symons, F.J. Pain experience and expression in Rett syndrome: Subjective and objective measurement approaches. J. Dev. Phys. Disabil. 2015, 27, 417-429. [CrossRef] [PubMed]

18. Peebles, K.A.; Price, T.J. Self-injurious behaviour in intellectual disability syndromes: Evidence for aberrant pain signalling as a contributing factor. J. Intell. Disabil. Res. 2012, 56, 441-452. [CrossRef] [PubMed]

19. Motil, K.J.; Schultz, R.J.; Browning, K.; Trautwein, L.; Glaze, D.G. Oropharyngeal dysfunction and gastroesophageal dysmotility are present in girls and women with Rett syndrome. J. Pediatr. Gastroenterol. Nutr. 1999, 29, 31-37. [CrossRef] [PubMed]

20. Motil, K.J.; Caeg, E.; Barrish, J.O.; Geerts, S.; Lane, J.B.; Percy, A.K.; Annese, F.; McNair, L.; Skinner, S.A.; Lee, H.S.; et al. Gastrointestinal and nutritional problems occur frequently throughout life in girls and women with Rett syndrome. J. Pediatr. Gastroenterol. Nutr. 2012, 55, 292-298. [CrossRef] [PubMed]

21. Krizsan-Agbas, D.; Winter, M.K.; Eggimann, L.S.; Meriwether, J.; Berman, N.E.; Smith, P.G.; McCarson, K.E. Gait analysis at multiple speeds reveals differential functional and structural outcomes in response to graded spinal cord injury. J. Neurotrauma 2014, 31, 846-856. [CrossRef] [PubMed]

22. Bhattacherjee, A.; Mu, Y.; Winter, M.K.; Knapp, J.R.; Eggimann, L.S.; Gunewardena, S.S.; Kobayashi, K.; Kato, S.; Krizsan-Agbas, D.; Smith, P.G. Neuronal cytoskeletal gene dysregulation and mechanical hypersensitivity in a rat model of Rett syndrome. Proc. Natl. Acad. Sci. USA 2017, 114, E6952-E6961. [CrossRef] [PubMed]

23. Orefice, L.L.; Zimmerman, A.L.; Chirila, A.M.; Sleboda, S.J.; Head, J.P.; Ginty, D.D. Peripheral Mechanosensory Neuron Dysfunction Underlies Tactile and Behavioral Deficits in Mouse Models of ASDs. Cell 2016, 166, 299-313. [CrossRef] [PubMed]

24. Christianson, J.A.; Gebhart, G.F. Assessment of colon sensitivity by luminal distension in mice. Nat. Protoc. 2007, 2, 2624-2631. [CrossRef] [PubMed]

25. Cheng, N.; Rho, J.M.; Masino, S.A. Metabolic Dysfunction Underlying Autism Spectrum Disorder and Potential Treatment Approaches. Front. Mol. Neurosci. 2017, 10, 34. [CrossRef] [PubMed]

26. El-Ansary, A.; Bjorklund, G.; Chirumbolo, S.; Alnakhli, O.M. Predictive value of selected biomarkers related to metabolism and oxidative stress in children with autism spectrum disorder. Metab. Brain Dis. 2017, 32, 1209-1221. [CrossRef] [PubMed]

27. Mogil, J.S. Animal models of pain: Progress and challenges. Nat. Rev. Neurosci. 2009, 10, 283-294. [CrossRef] [PubMed]

28. Temudo, T.; Ramos, E.; Dias, K.; Barbot, C.; Vieira, J.P.; Moreira, A.; Calado, E.; Carrilho, I.; Oliveira, G.; Levy, A.; et al. Movement disorders in Rett syndrome: An analysis of 60 patients with detected MECP2 mutation and correlation with mutation type. Mov. Disord. 2008, 23, 1384-1390. [CrossRef] [PubMed]

29. Chen, R.Z.; Akbarian, S.; Tudor, M.; Jaenisch, R. Deficiency of methyl-CpG binding protein-2 in CNS neurons results in a Rett-like phenotype in mice. Nat. Genet. 2001, 27, 327-331. [CrossRef] [PubMed]

30. Guy, J.; Hendrich, B.; Holmes, M.; Martin, J.E.; Bird, A. A mouse Mecp2-null mutation causes neurological symptoms that mimic Rett syndrome. Nat. Genet. 2001, 27, 322-326. [CrossRef] [PubMed]

31. De Filippis, B.; Musto, M.; Altabella, L.; Romano, E.; Canese, R.; Laviola, G. Deficient Purposeful Use of Forepaws in Female Mice Modelling Rett Syndrome. Neural Plast. 2015, 2015. [CrossRef] [PubMed]

32. Naidu, S.; Bibat, G.; Kratz, L.; Kelley, R.I.; Pevsner, J.; Hoffman, E.; Cuffari, C.; Rohde, C.; Blue, M.E.; Johnston, M.V. Clinical variability in Rett syndrome. J. Child Neurol. 2003, 18, 662-668. [CrossRef] [PubMed]

33. Neul, J.L.; Lane, J.B.; Lee, H.S.; Geerts, S.; Barrish, J.O.; Annese, F.; Baggett, L.M.; Barnes, K.; Skinner, S.A.; Motil, K.J.; et al. Developmental delay in Rett syndrome: Data from the natural history study. J. Neurodev. Disord. 2014, 6, 20. [CrossRef] [PubMed]

34. Chin Wong, L.; Hung, P.L.; Jan, T.Y.; Lee, W.T.; Taiwan Rett Syndrome Association. Variations of stereotypies in individuals with Rett syndrome: A nationwide cross-sectional study in Taiwan. Autism Res. Off. J. Int. Soc. Autism Res. 2017, 10, 1204-1214. [CrossRef] [PubMed]

35. Marco, E.J.; Hinkley, L.B.; Hill, S.S.; Nagarajan, S.S. Sensory processing in autism: A review of neurophysiologic findings. Pediatr. Res. 2011, 69, 48R-54R. [CrossRef] [PubMed]

36. Riquelme, I.; Hatem, S.M.; Montoya, P. Abnormal Pressure Pain, Touch Sensitivity, Proprioception, and Manual Dexterity in Children with Autism Spectrum Disorders. Neural Plast. 2016, 2016. [CrossRef] [PubMed] 
37. Baikie, G.; Ravikumara, M.; Downs, J.; Naseem, N.; Wong, K.; Percy, A.; Lane, J.; Weiss, B.; Ellaway, C.; Bathgate, K.; et al. Gastrointestinal dysmotility in Rett syndrome. J. Pediatr. Gastroenterol. Nutr. 2014, 58, 237-244. [CrossRef] [PubMed]

38. Farmer, A.D.; Aziz, Q. Gut pain \& visceral hypersensitivity. Br. J. Pain 2013, 7, 39-47. [PubMed]

39. Kiba, T. Relationships between the autonomic nervous system, humoral factors and immune functions in the intestine. Digestion 2006, 74, 215-227. [CrossRef] [PubMed]

40. Tougas, G. The autonomic nervous system in functional bowel disorders. Can. J. Gastroenterol. 1999, 13 (Suppl. A), 15A-17A. [CrossRef] [PubMed]

41. Uesaka, T.; Young, H.M.; Pachnis, V.; Enomoto, H. Development of the intrinsic and extrinsic innervation of the gut. Dev. Biol. 2016, 417, 158-167. [CrossRef] [PubMed]

42. Mayer, E.A. Gut feelings: The emerging biology of gut-brain communication. Nat. Rev. Neurosci. 2011, 12, 453-466. [CrossRef] [PubMed]

43. Ramirez, J.M.; Ward, C.S.; Neul, J.L. Breathing challenges in Rett syndrome: Lessons learned from humans and animal models. Respir. Physiol. Neurobiol. 2013, 189, 280-287. [CrossRef] [PubMed]

44. Lioy, D.T.; Wu, W.W.; Bissonnette, J.M. Autonomic dysfunction with mutations in the gene that encodes methyl-CpG-binding protein 2: Insights into Rett syndrome. Auton. Neurosci. Basic Clin. 2011, 161, 55-62. [CrossRef] [PubMed]

45. Panighini, A.; Duranti, E.; Santini, F.; Maffei, M.; Pizzorusso, T.; Funel, N.; Taddei, S.; Bernardini, N.; Ippolito, C.; Virdis, A.; et al. Vascular dysfunction in a mouse model of Rett syndrome and effects of curcumin treatment. PLoS ONE 2013, 8, e64863. [CrossRef] [PubMed]

46. Fyffe, S.L.; Neul, J.L.; Samaco, R.C.; Chao, H.T.; Ben-Shachar, S.; Moretti, P.; McGill, B.E.; Goulding, E.H.; Sullivan, E.; Tecott, L.H.; et al. Deletion of Mecp2 in Sim1-expressing neurons reveals a critical role for $\mathrm{MeCP} 2$ in feeding behavior, aggression, and the response to stress. Neuron 2008, 59, 947-958. [CrossRef] [PubMed]

47. Samaco, R.C.; Fryer, J.D.; Ren, J.; Fyffe, S.; Chao, H.T.; Sun, Y.; Greer, J.J.; Zoghbi, H.Y.; Neul, J.L. A partial loss of function allele of methyl-CpG-binding protein 2 predicts a human neurodevelopmental syndrome. Hum. Mol. Genet. 2008, 17, 1718-1727. [CrossRef] [PubMed]

48. Ward, C.S.; Arvide, E.M.; Huang, T.W.; Yoo, J.; Noebels, J.L.; Neul, J.L. MeCP2 is critical within HoxB1-derived tissues of mice for normal lifespan. J. Neurosci. 2011, 31, 10359-10370. [CrossRef] [PubMed]

49. Zappella, M.; Meloni, I.; Longo, I.; Hayek, G.; Renieri, A. Preserved speech variants of the Rett syndrome: Molecular and clinical analysis. Am. J. Med. Genet. 2001, 104, 14-22. [CrossRef] [PubMed]

50. Segatto, M.; Trapani, L.; Di Tunno, I.; Sticozzi, C.; Valacchi, G.; Hayek, J.; Pallottini, V. Cholesterol metabolism is altered in Rett syndrome: A study on plasma and primary cultured fibroblasts derived from patients. PLoS ONE 2014, 9, e104834. [CrossRef] [PubMed]

51. Buchovecky, C.M.; Turley, S.D.; Brown, H.M.; Kyle, S.M.; McDonald, J.G.; Liu, B.; Pieper, A.A.; Huang, W.; Katz, D.M.; Russell, D.W.; et al. A suppressor screen in Mecp2 mutant mice implicates cholesterol metabolism in Rett syndrome. Nat. Genet. 2013, 45, 1013-1020. [CrossRef] [PubMed]

52. Iannitti, T.; Graham, A.; Dolan, S. Increased central and peripheral inflammation and inflammatory hyperalgesia in Zucker rat model of leptin receptor deficiency and genetic obesity. Exp. Physiol. 2012, 97, 1236-1245. [CrossRef] [PubMed]

53. Okifuji, A.; Hare, B.D. The association between chronic pain and obesity. J. Pain Res. 2015, 8, 399-408. [CrossRef] [PubMed]

54. Gigli, C.C.; Scaramuzza, L.; Gandaglia, A.; Bellini, E.; Gabaglio, M.; Parolaro, D.; Kilstrup-Nielsen, C.; Landsberger, N.; Bedogni, F. MeCP2 related studies benefit from the use of CD1 as genetic background. PLOS ONE 2016, 11, e0153473.

55. Vogel Ciernia, A.; Pride, M.C.; Durbin-Johnson, B.; Noronha, A.; Chang, A.; Yasui, D.H.; Crawley, J.N.; LaSalle, J.M. Early motor phenotype detection in a female mouse model of Rett syndrome is improved by cross-fostering. Hum. Mol. Genet. 2017, 26, 1839-1854. [CrossRef] [PubMed]

(C) 2017 by the authors. Licensee MDPI, Basel, Switzerland. This article is an open access article distributed under the terms and conditions of the Creative Commons Attribution (CC BY) license (http:/ / creativecommons.org/licenses/by/4.0/). 